\title{
Design of E - Mail Based on Embedded Network Terminal
}

$$
\text { Ping Wang }{ }^{1, a} \text { and FuLan } \mathrm{Ye}^{2, \mathrm{~b}}
$$

${ }^{1}$ Information system, Fuzhou College of foreign studies and trade, Fuzhou, China, 350202

${ }^{2}$ Information system, Fuzhou College of foreign studies and trade, Fuzhou, China, 350202

apwang@fjnu.edu.cn, b466477987@qq.com

\section{Keywords: Embedded; Socket; E-mail; Chat software; WinCE}

\begin{abstract}
Aiming at the hardware systems and operating systems of embedded network terminal, solutions for chat software and E-mail client software based on embedded network terminal were proposed. In this paper, the design of chat software and E-mail client software were described in detail, after analyzing the network socket programming, the E-mail system theory and related protocols.
\end{abstract}

\section{Introduction}

At present, the embedded network application technology has become a very popular application technology in the international. In order to make a variety of devices have easy access to internet and achieve remote communications and control, Philips, Samsung and other companies are researching corresponding embedded network terminals. Because of their software and hardware resources are limited. Aiming at these problems, ARM + LAN chip was proposed. Coming with the operating system features and arm chip integrating most peripheral circuits, this model has fast speed, powerful functions ${ }^{[1]}$ and can provide a good hardware platform for the realization of internet applications.

E-mail client system and chat software are popular software for people exchanging information. Currently, although there are much such software, which only suitable for PC, but not for embedded network devices with limited resource, that designing chat software and email client software based on embedded network terminal, have high practical value.

\section{Network Terminal Hardware and Operating Systems}

Network Terminal Hardware System. Embedded network terminal was composed by S3C2440A-40 microprocessor of Samsung, the power module, Ethernet module, serial module, display modules (touch screen), external data storage module, system operation instructions module and interface modules.

Embedded Operating System. Developed by Microsoft, Windows CE operating system is a 32-bit embedded real time operating system with powerful functions, such as multi-threaded, multi-tasking, fully preemptive features, and is designed for a variety of hardware system with strict resource constraints ${ }^{[2]}$. Windows CE supports multiple hardware peripherals and makes users have easy access to internet to send or receive e-mail, browse web with built-in communications capabilities. In addition, the user interface of Windows CE system, which is similar to Windows system, makes people use easily ${ }^{[3]}$. Chat software and email clients use C/S model and were edited by VS.C \#.

\section{Chat Software}

TCP Connection-Oriented Communication Process. Using streaming socket programming, the user should have a connection channel before sending data. The communication process is divided into three steps:

Server Monitoring: After being created and launched, the Server will monitor on the designated port and wait for client connections.

Client requests: Using streaming socket create a client which is used to send connection requests 
to the designated Server. To meet this requirement, the client socket must point out the address and port number of the designated Server before sending connection request ${ }^{[4]}$ : TcpClient tcpClient $=$ new TcpClient(); tcpClient. Connect (IPAddress. Parse (server_ip), Int32. Parse (server_Port)).

Confirming connection: When the server detects a connection request (listener. Pending () is ture), Service process is awakened and generates a new byte streaming socket (Socket socket $=$ listener. AcceptSocket ()) to connect with the client. The old socket keeps on monitoring service requests on the network.

The Design of Chat Software. Both of the software is programmed by streaming socket, the server and client of chat software are in the same interface. Follows are the commonly used commands of chat protocol: JOIN, GETLIST, TALK, SECRET, EXIT, OFF.

Server's Design. First of all, creating a server socket by socket TcpListener class: TcpListener listener $=$ new TcpListener (localaddr_ip, port). Secondly, making the Server state: listener. Start(). establishing an independent data reception thread for each user to deal with the received data and commands. Creating thread: Thread clientService $=$ new Thread(new ThreadStart(client. ServiceClient)).

Client's Design. Using TcpClient class to create a streaming socket (tcpClient) as a client and providing the connected server's IP address and port number to the function of Connect() by clicking the friend from friends list. If your friend is not online (the friend's server does not open or his/her server does not detect the connection request), you can't communicate with he/she. After connected successfully, the client can write data or commands into the network data stream by "Byte[]bytes = System. Text. Encoding. Default. GetBytes();Stream. Write(bytes, 0, bytes. Length)".

Send and receive files by a pair of independent of server and client socket and using FileGetStream() to get the file's stream when sending files.

Major steps of sending file:

FileStream File = new FileStream (FilePath, FileMode. Open); //build file's stream object

byte[] by = new byte[System.Convert.ToInt32(File. Length)];//get the file's length

File. Read(by, 0, by. Length); //get the file's stream

Major steps of receive file:

filestream $=$ new

FileStream(saveFileDialog1.FileName,FileMode.OpenOrCreate,FileAccess.Write); //create a new file's stream;

while(filelength)//file's remaining length \{

filestream. Write(bytes, 0 , filelength);

filestream. Flush();

$\ldots . .$.

\section{E-Mail Client'S Design}

SMTP And POP3. SMTP(Simple Mail Transfer Protoco1) defines the rules of which communication of sending e-mail from source address to destination. Its commands are composed by ASCII and it provides 8 bytes transmission channel, the maximum bit is filled by zero.

The communication process of POP3 (Post Office Protocol) consists of three stages: verification state, transaction state and updating $\operatorname{state}^{[5]}$. Follows are the commonly used commands: DELE(delete e-mail), RETR + the e-mail's number (read e-mail), TOP (read specified e-mail header )and so on.

System's Design. As shown in Figure 1, RFC822 standard specifies syntax for text messages and applies only to the format and some of the semantics of message contents. To solve the problem of sending other kinds of information in e-mail, which include text in languages other than English using character encodings other than ASCII, and 8-bit binary content such as files containing images, sounds, movies, and computer programs, the design uses Multipurpose Internet Mail Extensions(MIME) standard. Commonly used encodings in the standard of MIME are 7 bit, 
quoted-printable, base64, 8 bit, binary.

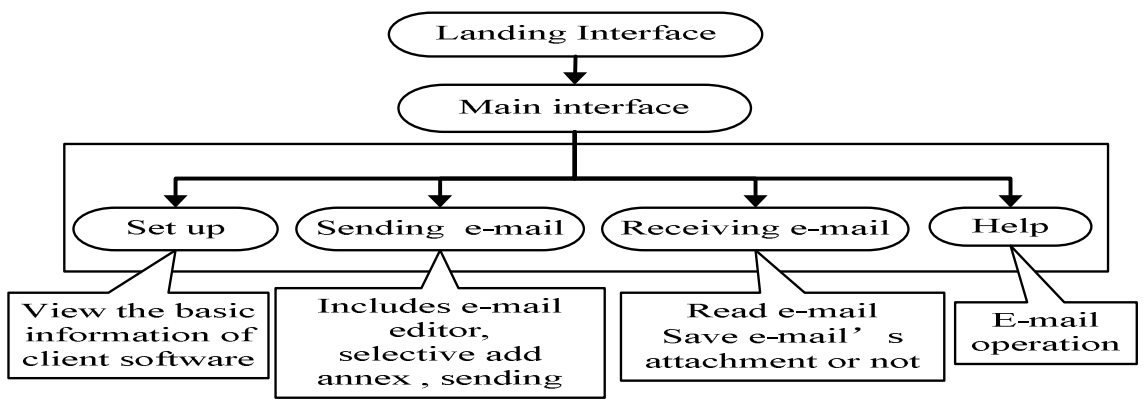

Figure 1. Main Frame

The Design of Sending E-Mail. As shown in Figure 2, the main functions of sending e-mail are Base64Encode(), SendCommand(), ReceiveData(), GetStream(), SendEmai(). Base64Encode()'s function is to make the sent string message into the format base64 encoding; SendCommand()'s function is to send a single commands; ReceiveData()'s function is to receive response code from STMP Server; GetStream()'s function is to get the attachment file's stream.

If the e-mail doesn't contain attachments, the client socket will send the e-mail directly, but if contains, the client socket will divide the e-mail's body into several parts: firstly, signs the Content-Type as multipart/mixed; secondly, defines the attribute of boundary which is a sub-identifier. The attachment could be voice files, images files and so on.
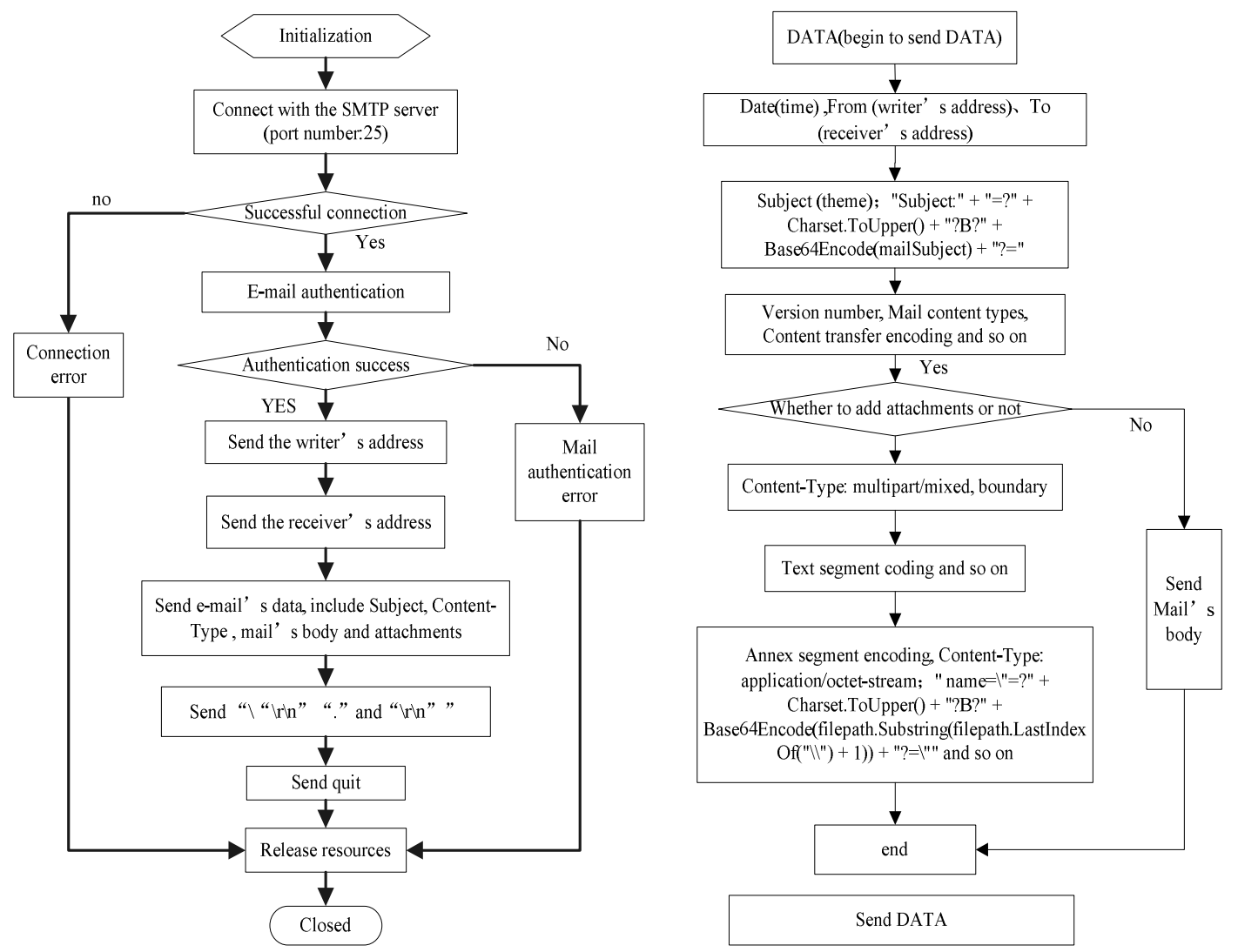

Figure 2. Sending E-mail Processes

The Design of Receiving E-Mail. As shown in Figure 3, combining the commands of POP3 protocol, Socket and NetworkStream, the client socket can get e-mail source code easily. But how to get the value message from the source code and convert them into the information that people can read are difficult. The e-mail reception system mainly rely on the classes of WincePOPClient, QUOTED-PRINTABLE, BASE64, SaveFileAttachment. 
The class of WincePOPClient mainly includes commands of POP3, the way of getting and analyzing mail source code and so on. Combining with the class of QUOTED-PRINTABLE, BASE64, the class of WincePOPClient can display the value information rightly. When watching or saving the attachment, the class of WincePOPClient will get the attachment source code from the class of SaveFileAttachment.

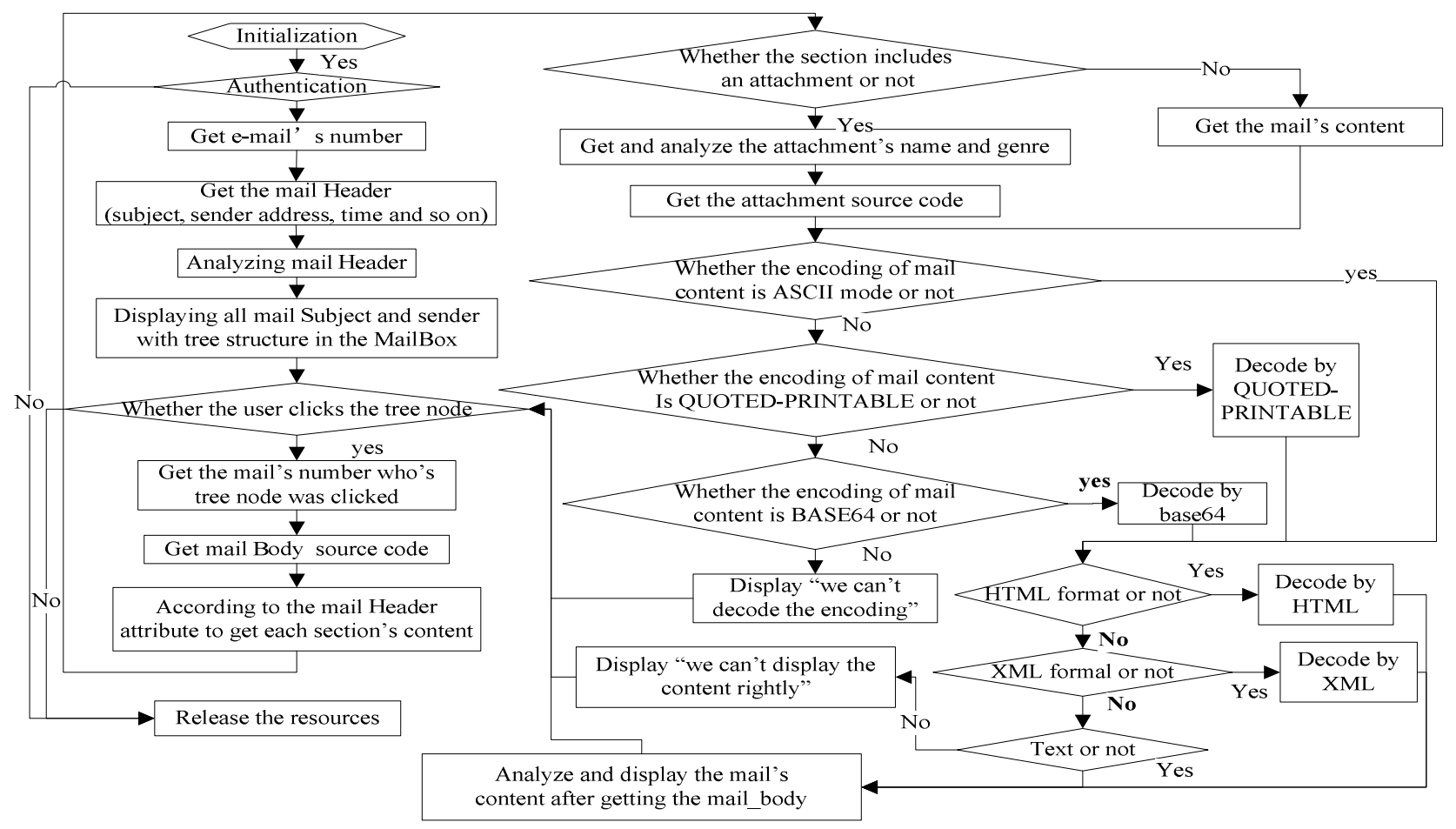

Figure 3. Receiving E-mail Processes

\section{Conclusions}

Based on the combination of network resource and the hardware platform of arm + LAN chip mode, the paper designs a chat software and e-mail client system in the Windows CE embedded operating system. E-mail client system solves the problem of decoding successfully. At the some time, the system is reliable, has a good user interface and the most common e-mail system functions. Both software meet the requirements of embedded system hardware successfully and satisfies the need of interaction, distribution and remote office, which make people chat, send and receive message easily by this network terminal.

\section{Acknowledgements}

This work was financially supported by the Fujian Provincial Department of Education Natural Science Foundation "Research on Portable Embedded Network Terminal" (F5039).

\section{References}

[1] Ping Wang: Microcomputer Applications, (2009) No. 1, p. 51 (In Chinese)

[2] Yimin Wu, Haijiang Ge and Nenggui Zhang: Mechanical and Electrical Engineering Magazine, (2008) No.9, p.10 (In Chinese)

[3] Jianmin Lin: Computer Engineering, (2001) No.10, p.1 (In Chinese)

[4] Qiaoling Li: Fujian Computer, (2009) No. 4, p. 135 (In Chinese)

[5] Shuhua Ye and Zhihong Gao: POSTS and TELECOM PRESS, (2006) No.1, p. 328(In Chinese) 